\title{
Compact First-Order Probe for Spherical Near-Field Antenna Measurements at Low Frequencies
}

\author{
Kim, Oleksiy S.
}

Published in:

I E E E Transactions on Antennas and Propagation

Link to article, DOI:

10.1109/TAP.2017.2700051

Publication date:

2017

Document Version

Peer reviewed version

Link back to DTU Orbit

Citation (APA):

Kim, O. S. (2017). Compact First-Order Probe for Spherical Near-Field Antenna Measurements at Low Frequencies. I E E E Transactions on Antennas and Propagation, 65(7), 3684 - 3690.

https://doi.org/10.1109/TAP.2017.2700051

\section{General rights}

Copyright and moral rights for the publications made accessible in the public portal are retained by the authors and/or other copyright owners and it is a condition of accessing publications that users recognise and abide by the legal requirements associated with these rights.

- Users may download and print one copy of any publication from the public portal for the purpose of private study or research.

- You may not further distribute the material or use it for any profit-making activity or commercial gain

- You may freely distribute the URL identifying the publication in the public portal 


\title{
Compact First-Order Probe for Spherical Near-Field Antenna Measurements at Low Frequencies
}

\author{
Oleksiy S. Kim
}

\begin{abstract}
Guidelines for designing compact and lightweight first-order probes for spherical near-field (SNF) antenna measurements at frequencies below $1 \mathrm{GHz}$ that exploit first-order properties of electrically small self-resonant radiators combined into superdirective endfire arrays are established theoretically, exemplified numerically, and validated experimentally. A prototype of the probe designed to operate at $435 \mathrm{MHz}$ central frequency exhibits the impedance bandwidth of $15 \mathrm{MHz}$ with more than $9 \mathrm{dBi}$ directivity and parasitic $|\mu| \neq 1$ spherical modes suppressed to at least $-42 \mathrm{~dB}$. The probe height is just $343 \mathrm{~mm}$ above a $720 \mathrm{~mm}$ circular ground plane and it weighs about $5 \mathrm{~kg}$.
\end{abstract}

Index Terms-Spherical near field, antenna measurements, electrically small antennas, supergain antennas, linear arrays, frequency bandwidth, physical bounds, quality factor, $Q$ factor, radiation

\section{INTRODUCTION}

$\mathbf{O}$ $\mathrm{NE}$ of the key elements in spherical near-field (SNF) antenna measurements is a probe - an antenna that detects the fields radiated by an antenna under test (AUT). As the name of the measurement technique suggests, the acquisition is carried out on a sphere in the near-field of the AUT. The recorded fields are expanded in a series of spherical waves, which are then propagated to the far-field where they are again combined into the AUT far-field radiation pattern. If the probe was a point source (Hertzian dipole), the procedure would be pretty straightforward. In real measurements, the probe is always of finite size implying that the signal on its output ports is a function of both the AUT radiated fields and the probe radiation pattern. To separate one from another, the near-to-far-filed transformation (NFFT) procedure must account for the probe influence. This is called "probe correction" [1].

From the point of view of complexity of both the measurement and the NFFT procedure as well as in terms of the resulting measurement accuracy, probes restricted to radiate only spherical modes with azimuthal indices $\mu= \pm 1$ are always preferable. This is why the vast majority of the probes found in SNF facilities around the world are openended cylindrical waveguides and conical horns excited by the fundamental mode $\mathrm{TE}_{11}$. This approach however faces its limitations at frequencies below $1 \mathrm{GHz}$, where classical probes become too bulky and heavy to be handled by a single person without lifting tools.

This work is supported by the European Space Agency (ESA) under ESA Contract no. 4000111981/15/NL/MH.

The author is with the Department of Electrical Engineering, Electromagnetic Systems, Technical University of Denmark, DK-2800 Kgs. Lyngby, Denmark (e-mail: osk@elektro.dtu.dk)
A lightweight and compact alternative to legacy probes was suggested in [2], where a superdirective linear array of two electrically small magnetic dipole elements was shown to be a viable solution for SNF measurements at low frequencies. The new probe exploits the cut-off property of spherical waves [1] that limits the mode spectrum of an electrically small source to dipole modes - the lowest-order $\mu= \pm 1$ modes. Superdirectivity allows the two-element array to yield more than $9 \mathrm{dBi}$ directivity. The superdirective probe developed for validation of another, wide-band higher-order 0.4-1 GHz probe [3] exhibited desirable first-order characteristics, although effectively at a single frequency, which nevertheless sufficed the purpose. To be compelling for general use, a superdirective probe should however provide much wider bandwidth, comparable to that of classical probes ${ }^{1}$.

In this paper, guidelines for designing superdirective probes with excellent first-order characteristics and improved impedance bandwidth are explained theoretically, illustrated numerically and validated experimentally. They have been implemented in a new compact first-order probe design, which has been fabricated and measured, providing a more than tenfold improvement in the bandwidth over the first prototype presented in [2]. While both probes represent a new class of probes for SNF antenna measurements - superdirective first-order probes - the one in [2] was built for a very specific task and was used only once, while the new probe was designed for regular use at the DTU-ESA SNF Antenna Test Facility [4] extending its operational frequency range covered by the facility's collection of first-order probes (conventional conical horns and open-ended cylindrical waveguides) to the lower UHF-band.

Preliminary results of this work were presented at a conference [5].

\section{ImPROVING THE BANDWIDTH OF A SUPERDIRECTIVE LiNEAR ARRAY of Dipole RADiators}

To find ways of extending the bandwidth of superdirective probes, we take a closer look at the theory of superdirective linear arrays of dipole radiators. The radiation quality factor $Q$ of such an array with maximum radiation in the endfire direction reads as

$$
Q \approx \frac{2}{3} Q_{e} Q_{g},
$$

where $Q_{e}$ is the radiation quality factor of an individual dipole radiator and $Q_{g}$ is the "geometrical" $Q$-factor expressing

\footnotetext{
${ }^{1}$ The operational bandwidth of open-ended cylindrical waveguides and conical horns is limited by the cut-off frequency of the $\mathrm{TM}_{01}$ mode. In practice, the useful bandwidth is about $11 \%$.
} 
"the influence which the geometrical arrangement exerts upon the bandwidth" [6]. Expression (1) is derived neglecting the mutual coupling between the array elements and assuming that $Q_{e}$ is large enough so that it can be approximated by the frequency derivative of the element's self-impedance. Despite being approximate, expression (1) immediately suggests how the bandwidth of a superdirective array can be improved - by extending the bandwidth of individual array elements (reducing $Q_{e}$ ) and by manipulating the geometry of the array to reduce $Q_{g}$.

In our case, individual array elements are in fact electrically small self-resonant magnetic dipole antennas [2], and thus the $Q$ theory of electrically small antennas applies here. In particular, the Chu lower bound on $Q$ [7] is inversely proportional to the cube of the electrical size of an antenna

$$
Q_{\mathrm{Chu}}=\frac{1}{(k a)^{3}}+\frac{1}{k a}
$$

where $k$ is the free-space propagation constant and $a$ is the radius of the minimum sphere circumscribing the antenna. It is therefore desirable to increase the size of the array elements. They cannot however be arbitrarily large, because the parasitic spherical modes with indices $|\mu|>1$ compromising the firstorder properties of the array increase accordingly. This is discussed in more details in the next section.

Further bandwidth improvement can be gained by efficiently utilizing the volume surrounding an electrically small antenna. For example, being effectively thin disks in shape, the capacitively loaded loops (CLL) used as array elements in [2] are inherently inferior in terms of $Q$ to volumetric magnetic dipole antennas of the same size. This comes from the fact that the lower bound on $Q$ for a thin disk is about 2.2 times higher than that for a spherical magnetic dipole of the same radius in free space $\left(6.7 Q_{\mathrm{Chu}}\right.$ and $3.0 Q_{\mathrm{Chu}}$, respectively [8]; both values are for $k a=0.1)$; the potential bandwidth is therefore 2.2 times narrower. For a cylindrical magnetic dipole antenna in free space with the moment along the cylinder axis and the optimal length to diameter ratio of 0.44 , the lower bound on $Q$ is 1.7 times lower than that for a thin disk $\left(4.0 Q_{\mathrm{Chu}}\right.$ and $6.7 Q_{\mathrm{Chu}}$, respectively [8]; both values are for $k a=0.1$ ). In other words, 3-D electrically small elements are always superior to 2-D elements of the same size in terms of the potential bandwidth.

If we had a low-loss highly permeable magnetic material available at our frequencies of interest, we would be able to reduce the $Q$ and improve the bandwidth of 3-D magnetic dipole elements even more by reducing the stored energy within their volumes [9]-[11]. The approach has also been shown theoretically feasible for individual electric dipole elements [12], [13] and superdirective linear arrays of those [14].

Finally, we will look at the arrangement of the elements within the array. Since the array is linear, the only parameter we can vary is the distance between the elements. As shown in [2], the endfire directivity of an array of $N$ dipole radiators arranged equidistantly along a normal to an infinite perfectly electrically conducting (PEC) ground plane, with their dipole moments parallel to each other and to the ground plane, and

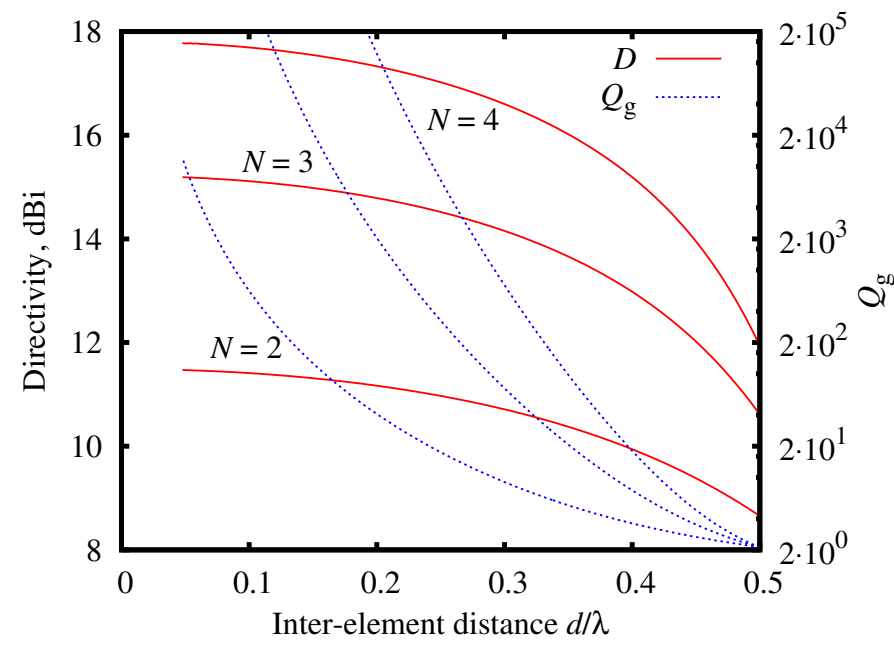

Fig. 1. Maximum attained endfire directivity and the corresponding "geometrical" $Q$ versus distance $d$ between the elements in linear arrays of two, three and four dipoles above an infinite PEC ground plane. The distance $d$ is normalized to the wavelength $\lambda$.

with the first element residing on the ground plane, is

$$
D=2 \sum_{n=0}^{N-1} b_{n} \cos (n k d)
$$

where $d$ is the distance between the dipoles. The directivity in (3) maximizes when the dipole excitation coefficients $b_{n}$ satisfy the following system of linear equations

$$
\sum_{n=0}^{N-1} b_{n} h_{m n}=\cos (m k d), \quad m=0,1, \ldots N-1
$$

where

$$
\begin{aligned}
& h_{m n}= \frac{1}{2} \sum_{ \pm}\left\{\frac{\sin [(m \pm n) k d]}{(m \pm n) k d}\left(1-\frac{1}{[(m \pm n) k d]^{2}}\right)\right. \\
&\left.+\frac{\cos [(m \pm n) k d]}{[(m \pm n) k d]^{2}}\right\}, \quad \text { for } m \neq n \\
& h_{m n}= \frac{1}{3}+\frac{\sin (2 n k d)}{4 n k d}\left(1-\frac{1}{(2 n k d)^{2}}\right)+\frac{\cos (2 n k d)}{2(2 n k d)^{2}}, \\
& \text { for } m=n \neq 0
\end{aligned}
$$

and $h_{m n}=2 / 3$ for $m=n=0$.

Another expression involving the excitation coefficients $b_{n}$ allows us to compute the "geometrical" $Q$ as [6]

$$
Q_{g}=\frac{\sum_{n=0}^{N-1} b_{n} b_{n}^{*}}{\sum_{n=0}^{N-1} \sum_{m=0}^{N-1} b_{n} h_{m n} b_{m}^{*}} .
$$

The maximum attained directivity and the corresponding "geometrical" $Q$ are plotted in Fig. 1 as a function of distance $d$ between the elements in arrays of two, three and four dipoles. With decreasing $d$, the directivity approaches its peak value for a given number of elements in the array, while the "geometrical" $Q$ tends to infinity, implying a vanishing bandwidth as $d \rightarrow 0$. The plot is a good illustration of superdirectivity and penalties associated with it. At the same 
time, it clearly shows that given a directivity value, the bandwidth can be maximized by selecting the largest possible interelement distance $d$. Weaker sensitivity to various errors (such as fabrication tolerances, misalignments, component parameter variations, etc.) and losses comes as an additional bonus [6].

To summarize the above findings, the bandwidth of a superdirective linear array of electrically small resonant elements can be improved in the following ways:

1) Enlarge the elements.

2) Use 3-D elements.

3) Increase the distance between the elements.

\section{NUMERICAL EXAMPLES}

To illustrate the theoretical conclusions of the previous Section, we consider resonant CLL's modelled as thin wires above an infinite PEC ground plane. First, we study properties of a single CLL, then, we modify it to make a 3-D element, and finally, investigate two of these in a two-element endfire array. The characteristics of interest are frequency bandwidth, relative radiated power of parasitic $|\mu| \neq 1$ modes, and directivity, in the case of the array.

All simulations were carried out with an in-house code solving a surface integral equation [15]. Every point in each plot in this Section was obtained for a corresponding antenna geometry tuned to resonance at $435 \mathrm{MHz}$ and input impedance of $50 \mathrm{ohms}$.

\section{A. Single CLL}

The geometry of a single CLL excited by curved monopoles above an infinite PEC ground plane is sketched in Fig. 2. If electrically small, this element radiates only dipole modes, owing to the cut-off property of spherical waves [1], and its spherical mode radiation spectrum thus contains only $\mu=0$ and $|\mu|=1$ spherical modes. When this element was used as an array element in [2], it was fed through port 1, while port 2 was short-circuited. Although the second monopole was passive, the symmetry of the structure effectively suppressed the modes with even azimuthal indices $\mu$, but only to a certain radius of the loop $r_{l} \approx 0.085 \lambda$, above which the relative radiated power of the parasitic $\mu=0$ modes started exceeding the maximum acceptable level of $-35 \mathrm{~dB}$. This is because the symmetry was merely enforced in the geometrical sense, while the induced currents had asymmetries that worsened as the element became larger.

If we want to use larger CLL's in our array, we need to enforce the symmetry not only in the geometrical but also in the electrical sense. This can be achieved by feeding port 2 $180^{\circ}$ out of phase with respect to port 1 . Enforcing the symmetry completely eliminates not only $\mu=0$ modes, but all the modes with even azimuthal indices $\mu$. The strongest parasitic modes left are then those with indices $|\mu|=3$.

Fig. 3 shows the fractional bandwidth at $-10 \mathrm{~dB}$ level and the relative radiated power of $|\mu|=3$ modes as a function of the loop radius $r_{l}$ normalized to the resonance wavelength at $435 \mathrm{MHz}$. As compared with the single-port element [2, Fig. 3], the radius of the dual-port CLL can be increased by $40 \%$, resulting in a more than doubled bandwidth, while holding all parasitic $|\mu| \neq 1$ modes below $-40 \mathrm{~dB}$.

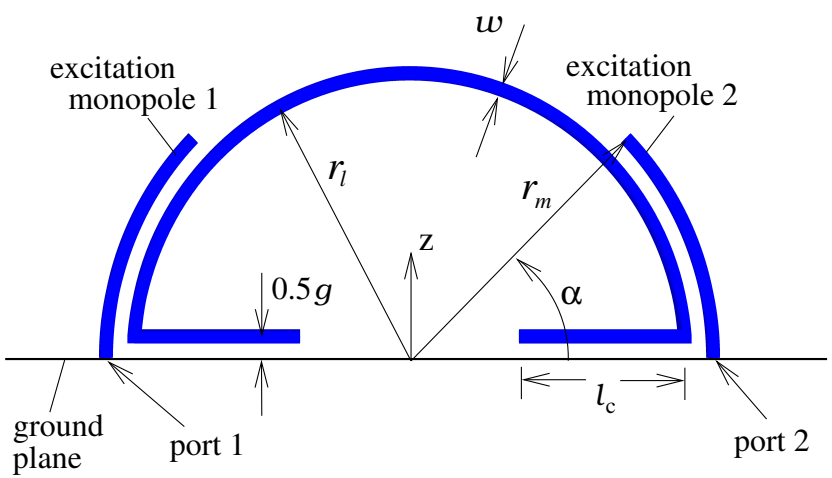

Fig. 2. Capacitively loaded loop (CLL) excited by curved monopoles above an infinite PEC ground plane. Diameter of the wires $w_{l}=1 \mathrm{~mm}$; capacitor gap $g=3 \mathrm{~mm}$; radius of the excitation monopoles $r_{m}=r_{l}+1.5 \mathrm{~mm}$. The length of the capacitor $l_{c}$ and the length of the excitation monopoles $\alpha$ are adjusted to tune the antenna to $50 \mathrm{ohms}$ at $435 \mathrm{MHz}$.

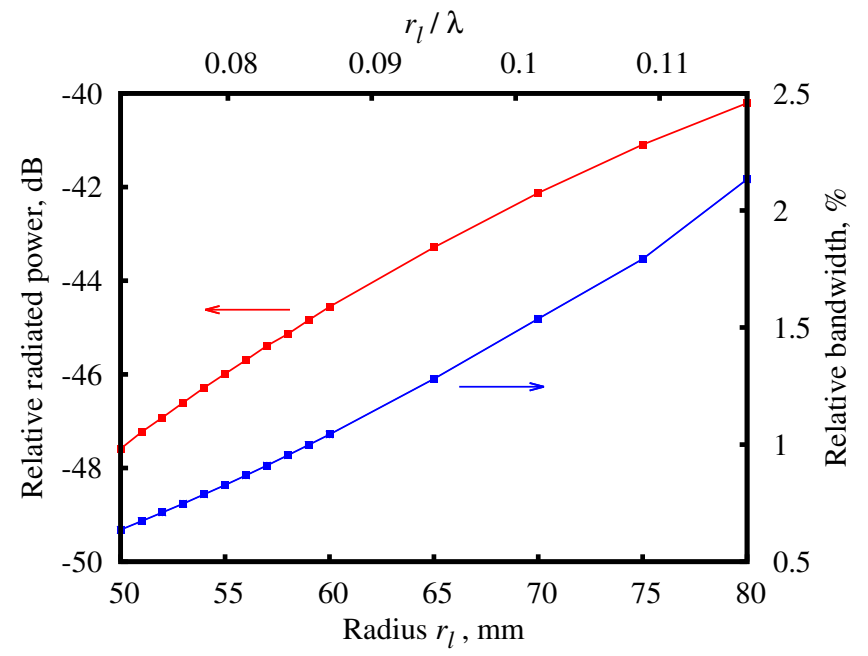

Fig. 3. Relative radiated power of $|\mu|=3$ spherical modes and fractional bandwidth at $-10 \mathrm{~dB}$ versus the radius of the CLL excited by two curved monopoles $180^{\circ}$ out of phase; see Fig. 2 . The directivity of the element grows linearly with $r_{l}$, from $5.1 \mathrm{dBi}$ to $5.8 \mathrm{dBi}$.

\section{B. 3-D CLL Element}

The bandwidth of the dual-port CLL can be further extended by properly developing the element in the axial direction. By adding co-axial passive CLL's on each side of the main active CLL, as illustrated in Fig. 4, we can create a cylindrical version of the spherical split ring antenna [16]. Since the three coupled loops act as a single 3-D self-resonant element, we can choose to redistribute the capacitive load to the outer loops by transforming the middle CLL into a simple split loop with the gap height $t$.

The results of the parametric investigation of the 3-D CLL element with the loop radii $r_{l}=70 \mathrm{~mm}$ are presented in Fig. 5, where the fractional bandwidth and the normalized radiated power of the $|\mu|=3$ modes are plotted versus the interloop distance $d_{y}$. The bandwidth exceeds that of the single CLL element (Fig. 3) at least by a factor of two, and steadily grows with $d_{y}$, owing to the corresponding expansion of the minimum sphere circumscribing the antenna.

Additional outer CLL's do not only improve the bandwidth, 


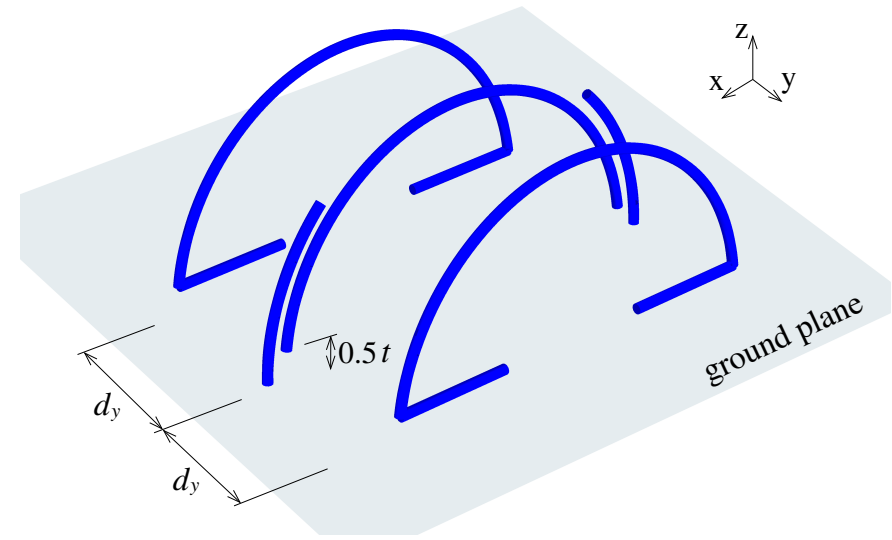

Fig. 4. Three coupled CLL's act as a single 3-D self-resonant element. The gap height $t=3 \mathrm{~mm}$. Other parameters are the same as in Fig. 2. The length of the capacitors $l_{c}$ and the length of the excitation monopoles $\alpha$ are adjusted to tune the antenna to $50 \mathrm{ohms}$ at $435 \mathrm{MHz}$.

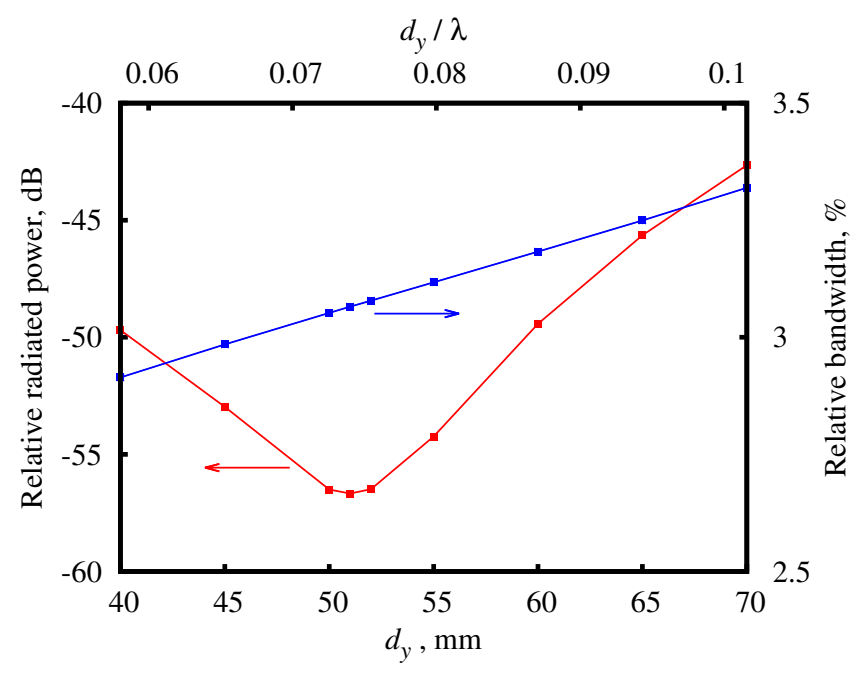

Fig. 5. Relative radiated power of $|\mu|=3$ spherical modes and fractional bandwidth at $-10 \mathrm{~dB}$ versus the separation between the loops $d_{y}$ in the 3-D CLL element (Fig. 4). For all three loops, $r_{l}=70 \mathrm{~mm}$. The directivity of the element is $5.8 \mathrm{dBi}$.

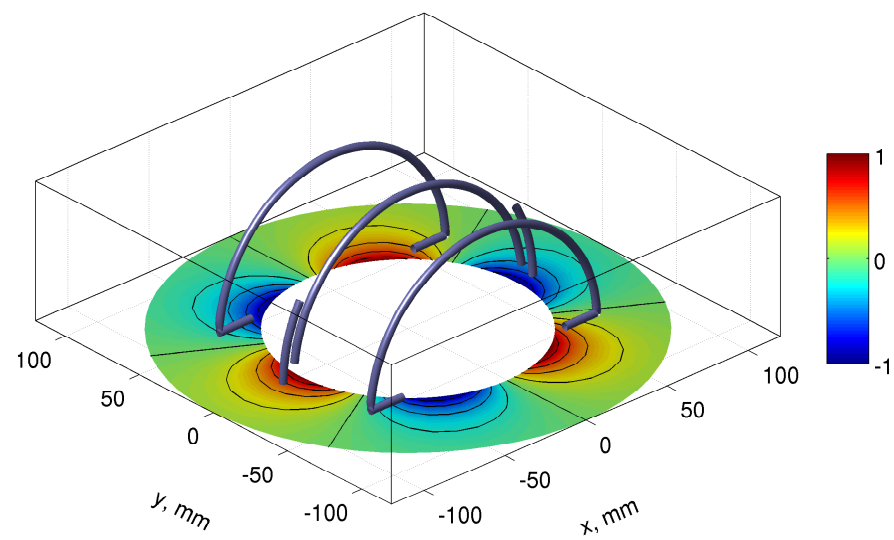

Fig. 6. Normalized amplitude distribution of the $\theta$-component of the $\mathrm{TE}_{33}$ spherical mode in the $x y$-plane $\left(\theta=90^{\circ}\right)$ relative to the three loops of the 3-D CLL element (Fig. 4).

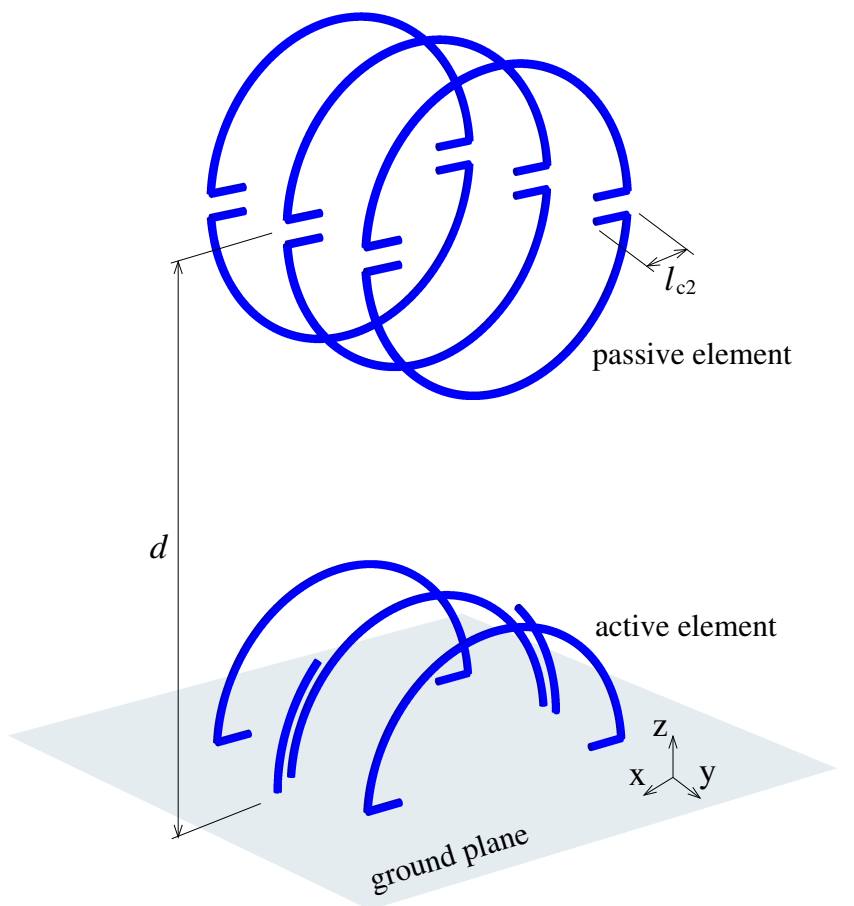

Fig. 7. A linear array of two 3-D CLL elements above a PEC ground plane. The active element is the same as in Fig. 4, while the passive element has its excitation monopoles removed and is offset by distance $d$. All three loops of the passive element are identical with the same capacitor length $l_{c 2}$. Other geometrical parameters are defined in Fig. 2 and Fig. 4.

they also effectively suppress the parasitic $|\mu|=3$ modes. We can understand the phenomenon by inspecting the distribution of the $\theta$-component of the electric field of the strongest parasitic $\mathrm{TE}_{33}$ mode $E_{\theta}^{\mathrm{TE}_{33}}$ in the $\theta=90^{\circ}$ plane. Fig. 6 shows the quantity $y_{3}(k r) / y_{3}\left(k r_{l}\right) \cos (3 \phi)$, which is proportional to $E_{\theta}^{\mathrm{TE}_{33}}$ [1], along with a sketch of the 3-D CLL element. $y_{3}(x)$ is the third-order spherical Bessel function of the second kind, and $r$ and $\phi$ are the spherical coordinates. To excite the $\mathrm{TE}_{33}$ mode, the currents on the outer CLL's should flow in the direction opposite to that of the current on the middle loop, whereas the actual currents are in phase with the current on the middle loop, which effectively suppresses the $\mathrm{TE}_{33}$ mode. Or, by reciprocity, the incoming $\mathrm{TE}_{33}$ mode excites the outer CLL's out of phase with the middle one, effectively cancelling the current on it, with the maximum cancellation occurring at some optimal separation between the loops $d_{y}$ (the optimal $d_{y}=51 \mathrm{~mm}$, in this particular case; see Fig. 5).

Similar considerations apply to other parasitic $|\mu|>1$ modes.

\section{Array of Two 3-D CLL Elements}

An array composed of two 3-D CLL elements, an active and a passive one, is sketched in Fig. 7. The active element is identical to that considered in Section III-B, while the passive element consists of three identical CLL's and is offset by distance $d$ from an infinite PEC ground plane. Although only one of the array elements is actively fed, the theory of superdirective arrays still applies, since both elements are selfresonant [17]. Alternatively, the array can be considered a 


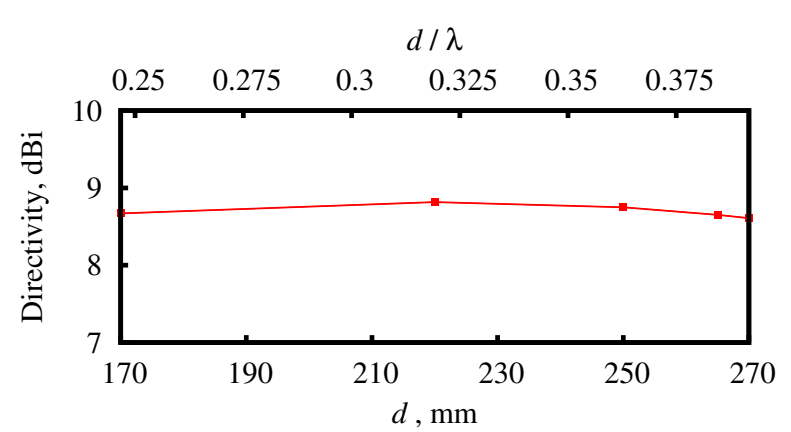

(a)

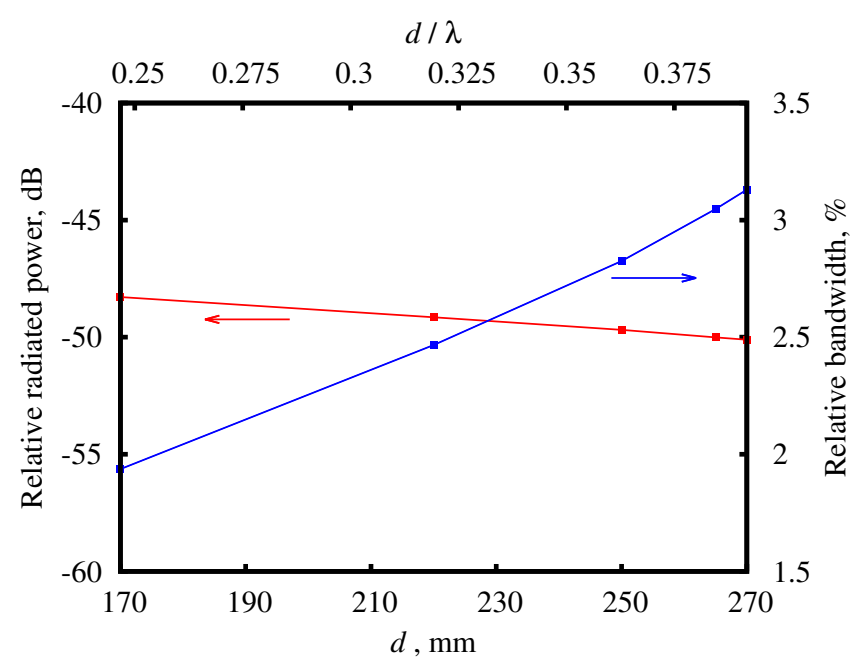

(b)

Fig. 8. Directivity (a), relative radiated power of $|\mu|=3$ spherical modes and fractional bandwidth at $-10 \mathrm{~dB}$ (b) versus distance $d$ between the elements in the two-element array (Fig. 7). For all the loops, $r_{l}=70 \mathrm{~mm}$. The distance between the loops in each element $d_{y}=57 \mathrm{~mm}$.

variation of a classical Yagi-Uda antenna, in which the passive element acts as a director.

Given an offset $d$, the directivity of the array is adjusted by properly phasing the passive element, via its capacitor length $l_{c 2}$, with respect to the active one. The array is then tuned to resonance at $435 \mathrm{MHz}$ and input impedance of $50 \mathrm{ohms}$ using the capacitor length $l_{c}$ of the active element and the length of the excitation monopoles $\alpha$. The results presented below have been obtained with $l_{c 2}$ set to $11 \mathrm{~mm}$, giving the directivity of about $8.5 \mathrm{dBi}$ for all considered values of $d$ (Fig. 8a). The radius of the loops was $r_{l}=70 \mathrm{~mm}$ and the inter-loop distance was $d_{y}=57 \mathrm{~mm}$.

The fractional bandwidth and the normalized radiated power of the $|\mu|=3$ modes are plotted in Fig. $8 \mathrm{~b}$ as a function of the distance $d$. While the level of the parasitic modes remains virtually unaffected, the bandwidth monotonically increases with the distance. As predicted by (1) and Fig. 1, the bandwidth of an array with closely spaced elements is narrower than the bandwidth of its individual elements due to a high "geometrical" $Q$, but gradually improves with increasing distance between the elements as the detrimental effect of the array weakens and the "geometrical" $Q$ decreases.

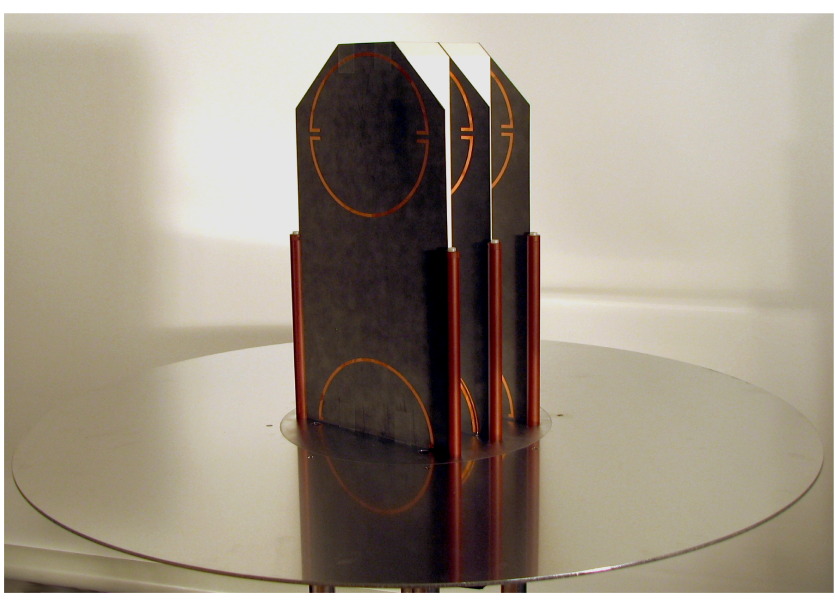

Fig. 9. Fabricated prototype of the improved P-band first-order probe. Main geometrical parameters are $r_{l}=70 \mathrm{~mm}, d=265 \mathrm{~mm}, d_{y}=57 \mathrm{~mm}$. Total height is $343 \mathrm{~mm}$, diameter of the ground plane is $720 \mathrm{~mm}$. Weight $5 \mathrm{~kg}$.

\section{P-BAnd First-Order Probe}

In the previous two sections, we have identified, and verified numerically on simplified models, several ways of extending the bandwidth of superdirective linear arrays of electrically small resonant elements. Based on these guidelines, an improved version of the superdirective first-order probe [2] has been designed. The design aimed at the directivity above $9 \mathrm{dBi}$ (to suppress specular reflections from the walls in the anechoic chamber [18]) in a frequency range of at least $435 \pm 3 \mathrm{MHz}$ (upcoming ESA Biomass mission frequency range [19]) with the parasitic $|\mu| \neq 1$ modes suppressed below $-35 \mathrm{~dB}$.

\section{A. Design}

Similar to [2], the CLL's are printed on a substrate, but instead of Rogers RO4003C laminate used in [2], Rogers $\mathrm{RT} /$ duroid 5880 is utilized in the new design, for its low dielectric constant and loss tangent. Since the distance $d_{y}$ between the loops in the active and the passive element is the same, the respective CLL's of both elements are coplanar, and therefore can be printed on the same substrate. Three substrates each containing two loops, one from the active element and another from the passive one, are arranged vertically, parallel to each other, separated by distance $d_{y}$, and centered on a circular aluminum ground plane. The substrates are supported at the edges by Tufnol rods and further reinforced by Rohacell blocks inserted in between. Two SMA connectors are attached to the bottom of the ground plane with their center pins protruding through the ground plane and soldered to the excitation monopoles printed on the middle substrate.

The fabricated probe is shown in Fig. 9. The total height of the probe is $343 \mathrm{~mm}$ above a $720 \mathrm{~mm}$ circular ground plane. The weight is about $5 \mathrm{~kg}$, mainly due to the ground plane.

\section{B. Results}

According to full wave simulations, the impedance bandwidth at $-10 \mathrm{~dB}$ level reaches $15 \mathrm{MHz}$ (Fig. 10b), which is an order of magnitude wider than the bandwidth of the superdirective probe presented in [2]. The directivity exceeds 


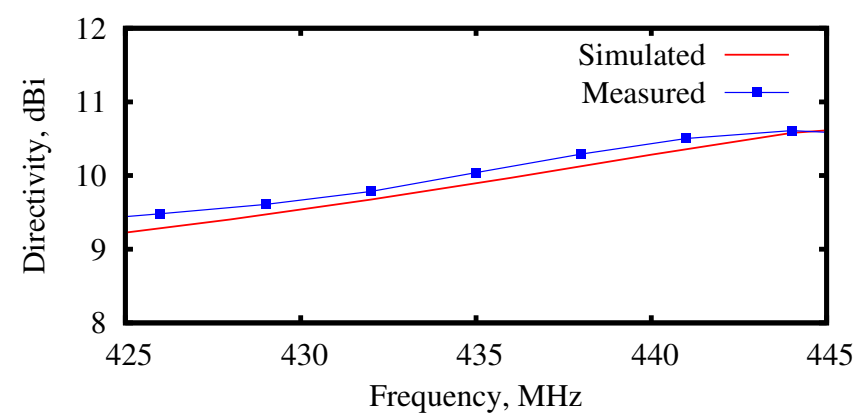

(a)

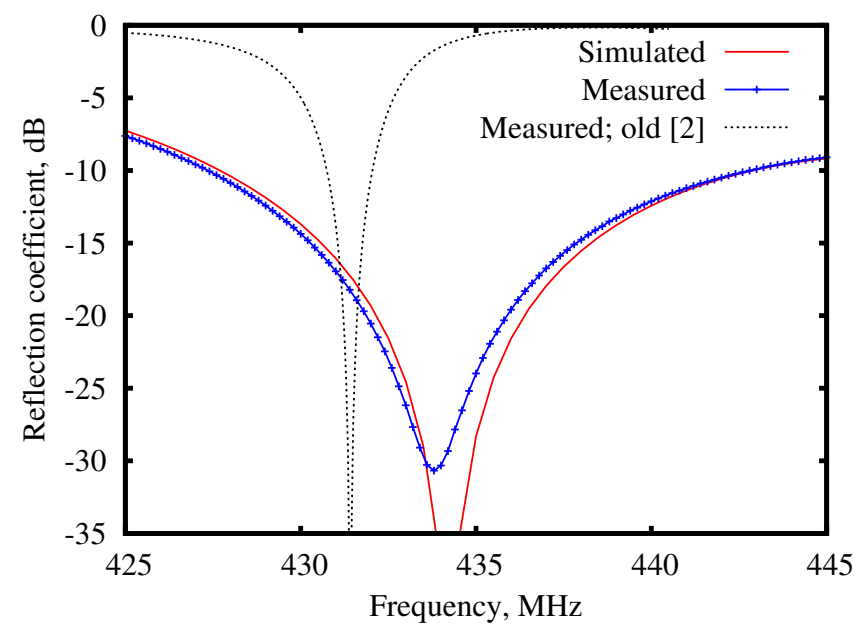

(b)

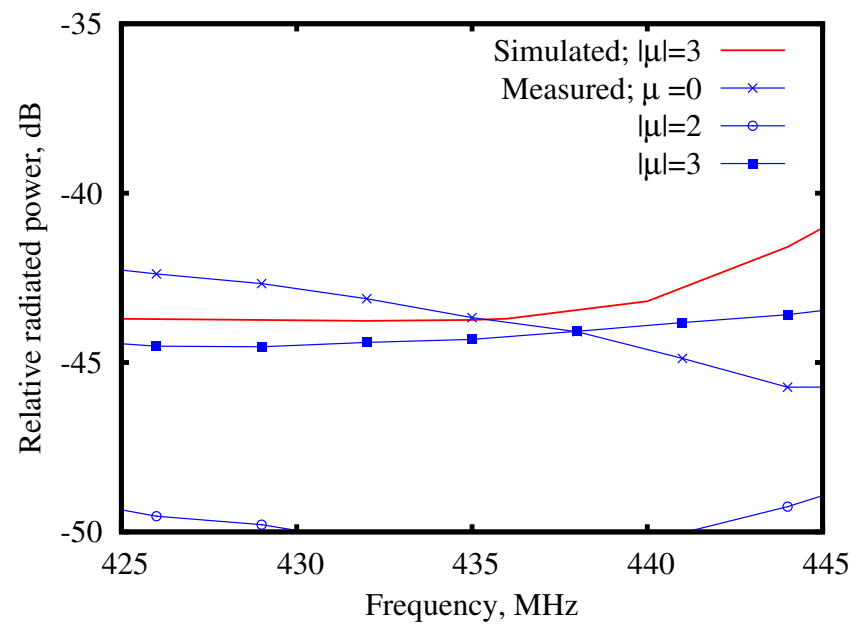

(c)

Fig. 10. Simulated and measured directivity (a), reflection coefficient (b), and relative radiated power of parasitic $|\mu| \neq 1$ spherical modes (c) versus frequency. The reflection coefficient for the new probe was computed assuming excitation by an ideal $180^{\circ}$ hybrid. The measured reflection coefficient of the first superdirective probe [2] is also plotted for reference.

$9 \mathrm{dBi}$ across the entire band (Fig. 10a), while the $|\mu|=3$ modes are suppressed to about $-44 \mathrm{~dB}$ (Fig. 10c). Due to the symmetry, all modes with even azimuthal indices, including $\mu=0$ and $|\mu|=2$ modes are way below $-50 \mathrm{~dB}$.

Measured results obtained at the DTU-ESA SNF Antenna Test Facility [4] are generally in close agreement with the simulations, although the measured reflection coefficient is slightly shifted towards lower frequencies (Fig. 10b). The
S-parameter measurements were carried out directly on the probe's two ports and the reflection coefficient was then computed assuming an ideal $180^{\circ}$ hybrid.

Besides $|\mu|=3$ modes, whose level is nearly as expected, $\mu=0$ modes are also present in the measured spherical mode spectra (Fig. 10c), indicating minor asymmetries in the fabricated probe structure as well as imbalances in the $180^{\circ}$ hybrid feeding the probe. Nevertheless, no $|\mu| \neq 1$ mode exceeds $-42 \mathrm{~dB}$ in the entire frequency range displayed in Fig. 10.

The results above show that the useful bandwidth of the probe is limited by its impedance, whereas the directivity and the purity of the $|\mu|=1$ modes allow the probe to be used in a wider frequency range, if one can tolerate higher than $-10 \mathrm{~dB}$ reflection coefficient. Alternatively, the useful bandwidth can be improved by applying an external matching circuit or even a simple attenuator.

Measured radiation patterns at $426 \mathrm{MHz}, 435 \mathrm{MHz}$, and $444 \mathrm{MHz}$ are shown in Fig. 11. The probe exhibits desirable radiation characteristics at all frequencies, with the amplitude variation not exceeding $5 \mathrm{~dB}$ within the AUT field of view $\left(\theta \leq \pm 30^{\circ}\right.$ for our anechoic chamber [4]), the front-to-back ratio greater than $15 \mathrm{~dB}$, and the on-axis cross polarization of $-40 \mathrm{~dB}$ or better. At frequencies above $445 \mathrm{MHz}$, the sidelobe level may become prohibitively high. On the other hand, one may still want to use the probe below $425 \mathrm{MHz}$, if one can cope with the high reflection coefficient and tolerate somewhat lower directivity.

\section{CONCLUSIONS}

The theories of superdirective linear arrays and electrically small antennas have been revisited to identify possible ways of achieving a reasonably wide impedance bandwidth for superdirective linear arrays of electrically small self-resonant magnetic dipole elements. Such an array can be used as a first-order probe in SNF antenna measurements at frequencies below $1 \mathrm{GHz}$, where conventional probes, such as open-ended cylindrical waveguides and conical horns, become too bulky and heavy. Established guidelines include

- enforcing the symmetry of the structure and the excitation to suppress spherical modes with even azimuthal indices;

- using volumetric array elements as large as the parasitic $|\mu|=3$ modes allow;

- enlarging the distance between the array elements.

Following the guidelines, a new probe was designed, fabricated and measured. It comprises two volumetric self-resonant magnetic dipole elements, each composed of three coaxial capacitively loaded loops (CLL's). These new elements do not only facilitate wider impedance bandwidth, but also effectively suppress $|\mu|>1$ spherical modes.

The probe exhibits excellent first-order characteristics with parasitic $|\mu| \neq 1$ spherical modes below $-42 \mathrm{~dB}$ and the directivity exceeding $9 \mathrm{dBi}$ across the entire impedance bandwidth of $15 \mathrm{MHz}$ (at $-10 \mathrm{~dB}$ level of reflection coefficient, with the central frequency at $435 \mathrm{MHz}$ ), which is a more than tenfold improvement over the first superdirective probe presented in [2]. The height of the new probe is about $0.5 \lambda$ above $1 \lambda$ circular ground plane; the weight is about $5 \mathrm{~kg}$. 


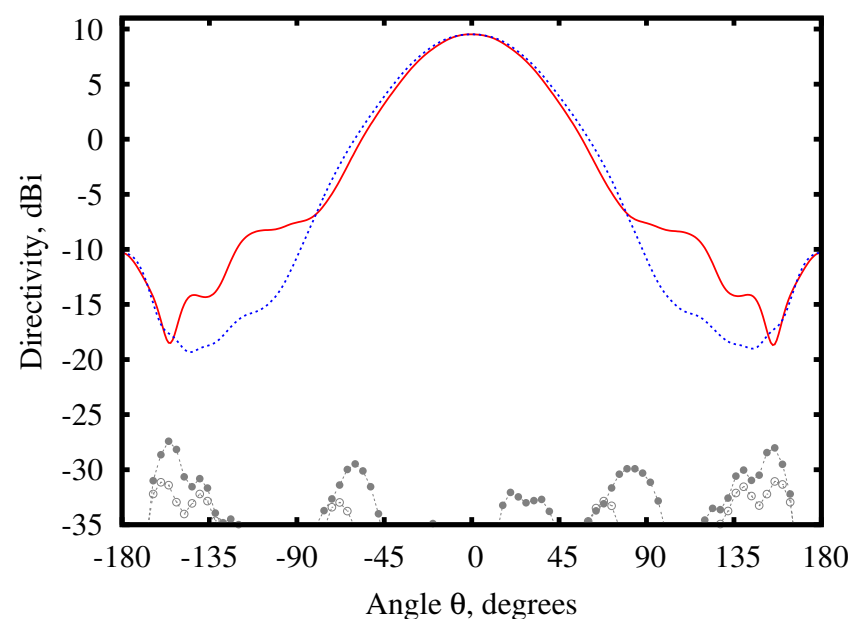

(a)

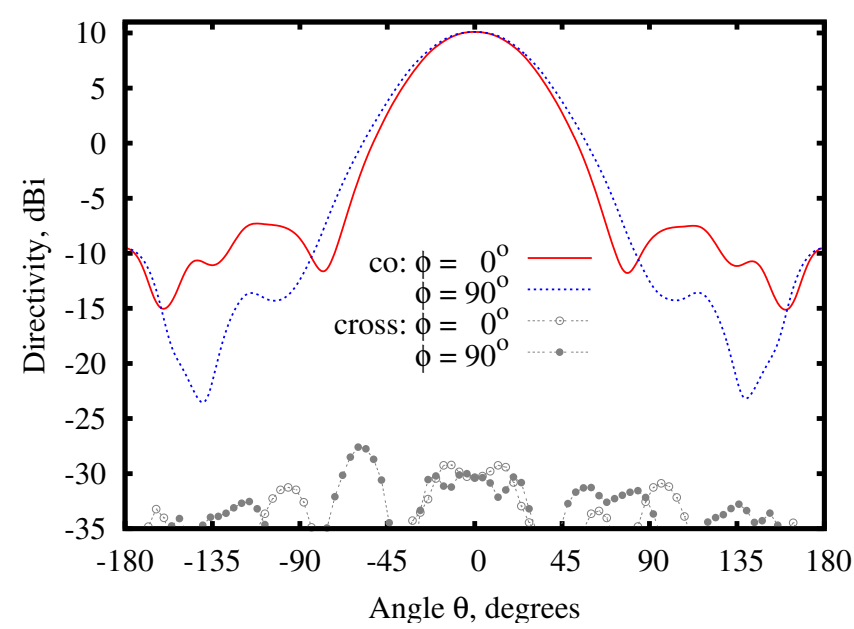

(b)

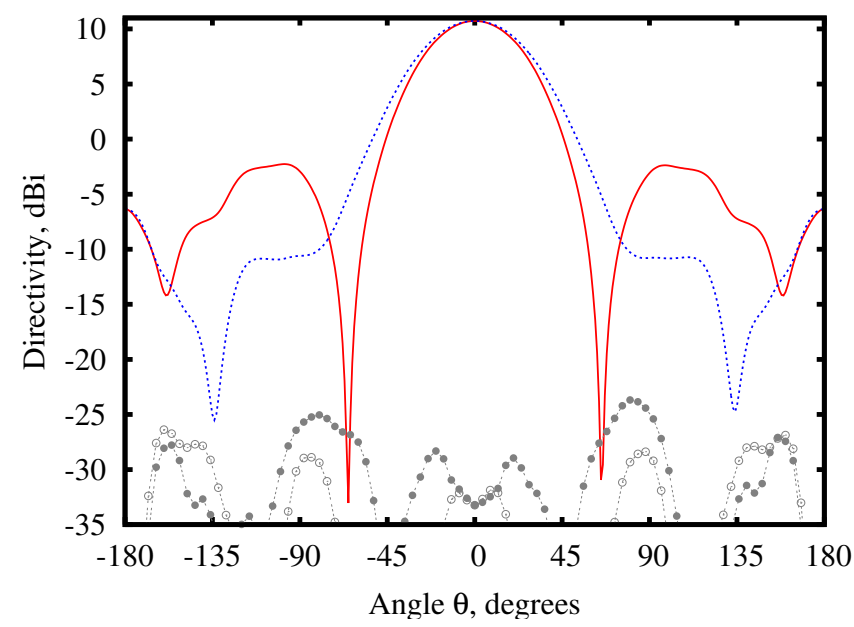

(c)

Fig. 11. Measured radiation patterns of the fabricated probe at $426 \mathrm{MHz}$ (a), $435 \mathrm{MHz}(\mathrm{b})$, and $444 \mathrm{MHz}$ (c).

Thus, a two-element linear array of electrically small selfresonant CLL's has proven to be a compact and lightweight alternative to open-ended cylindrical waveguides and conical horns when used as a first-order probe in SNF measurements at low frequencies.

\section{ACKNOWLEDGEMENT}

Martin Nilesen and Jan N. Mortensen are acknowledged for making parts for the probe and assembling it. Jeppe M. Nielsen is acknowledged for carrying out the radiation measurements.

\section{REFERENCES}

[1] J. E. Hansen, Spherical Near-Field Antenna Measurements. London, U.K.: Peter Peregrinus, 1988.

[2] O. S. Kim, S. Pivnenko, and O. Breinbjerg, "Superdirective magnetic dipole array as a first-order probe for spherical near-field antenna measurements," IEEE Trans. Antennas Propagat., vol. 60, no. 10, pp. 4670-4676, 2012.

[3] — " "0.4-1.2 GHz hybrid Al-CFRP open-boundary quad-ridge horn," in Proc. 33rd ESA Antenna Workshop, ESTEC, Noordwijk, the Netherlands, 18-21 October 2011.

[4] DTU-ESA Spherical Near-Field Antenna Test Facility. Homepage, http: //www.ems.elektro.dtu.dk/research/dtu_esa_facility.

[5] O. S. Kim, "Extending the bandwidth of a superdirective first-order probe for spherical near-field antenna measurements," in Proc. Int. Symp. Ant. Propagat. (ISAP), Hobart, Tasmania, Australia, 9-12 Nov 2015.

[6] M. Uzsoky and L. Solymar, "Theory of super-directive linear arrays," Acta Phys. Hungarica, vol. 6, no. 2, pp. 185-205, 1956.

[7] L. J. Chu, "Physical limitations of omni-directional antennas," J. Appl. Phys., vol. 19, no. 12, pp. 1163-1175, 1948.

[8] O. S. Kim, "Lower bounds on Q for finite size antennas of arbitrary shape," IEEE Trans. Antennas Propagat., vol. 64, no. 1, pp. 146-154, January 2016.

[9] O. S. Kim, O. Breinbjerg, and A. D. Yaghjian, "Electrically small magnetic dipole antennas with quality factors approaching the Chu lower bound," IEEE Trans. Antennas Propagat., vol. 58, no. 6, pp. 1898-1906, June 2010.

[10] O. S. Kim and O. Breinbjerg, "Lower bound for the radiation Q of electrically small magnetic dipole antennas with solid magnetodielectric core," IEEE Trans. Antennas Propagat., vol. 59, no. 2, pp. 679-681, February 2011.

[11] _ "Reaching the Chu lower bound on $\mathrm{Q}$ with magnetic dipole antennas using a magnetic-coated PEC core," IEEE Trans. Antennas Propagat., vol. 59, no. 8, pp. 2799-2805, August 2011.

[12] H. R. Stuart and A. D. Yaghjian, "Approaching the lower bounds on $\mathrm{Q}$ for electrically small electric-dipole antennas using high permeability shells," IEEE Trans. Antennas Propagat., vol. 58, no. 12, pp. 3865-3872, December 2010.

[13] O. S. Kim, "Electric dipole antennas with magnetic-coated PEC cores: Reaching the Chu lower bound on Q," IEEE Trans. Antennas Propagat., vol. 60, no. 3, pp. 1616-1619, 2012.

[14] A. D. Yaghjian and H. R. Stuart, "Increasing the bandwidth of electrically small supergain antennas using low-Q electric dipoles," in Proc. 5th European Conference on Antennas and Propagation (EuCAP'2011), Rome, Italy, April 2011, pp. 2720-2723.

[15] O. S. Kim, E. Jørgensen, P. Meincke, and O. Breinbjerg, "Higherorder hierarchical Legendre basis functions in applications," in The Fourth Swedish Conference on Computational Electromagnetics. Lund University, 2007, pp. 239-246.

[16] O. S. Kim, "Low-Q electrically small spherical magnetic dipole antennas," IEEE Trans. Antennas Propagat., vol. 58, no. 7, pp. 2210-2217, July 2010.

[17] A. D. Yaghjian, T. H. O'Donnell, E. E. Altshuler, and S. Best, "Electrically small supergain end-fire arrays," Radio Science, vol. 43, no. 3, p. RS3002, 2008.

[18] O. S. Kim, S. Pivnenko, and O. Breinbjerg, "Wideband scalable probe for spherical near-field antenna measurements," in Proc. 5th European Conference on Antennas and Propagation (EuCAP'2011), Rome, Italy, 2011, pp. $1781-1785$.

[19] F. Heliere, F. Fois, M. Arcioni, P. Bensi, M. Fehringer, and K. Scipal, "Biomass P-band SAR interferometric mission selected as 7th earth explorer mission," in Proc. 10th European Conf. Synthetic Aperture Radar EUSAR 2014, June 2014, pp. 1-4. 


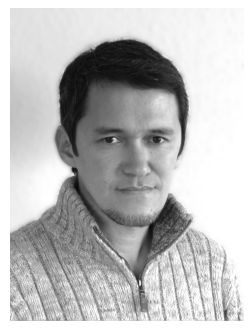

Oleksiy S. Kim received the M.Sc. and Ph.D. degrees from the National Technical University of Ukraine, Kiev, in 1996 and 2000, respectively, both in electrical engineering. In 2000, he joined the Antenna and Electromagnetics Group at the Technical University of Denmark (DTU). He is currently an associate professor with the Department of Electrical Engineering, Electromagnetic Systems, DTU.

His research interests include electrically small antennas, computational electromagnetics, metamaterials, photonic bandgap and plasmonic structures. 